\title{
Endometrial and cervical cancer patients with multiple sentinel lymph nodes at laparoscopic ICG mapping: How many are enough?
}

\author{
Andrea Papadia $^{1} \cdot$ Sara Imboden $^{1} \cdot$ Maria Luisa Gasparri $^{1,2}$. \\ Franziska Siegenthaler $^{1} \cdot$ Anja Fink $^{1} \cdot$ Michael D. Mueller $^{1}$
}

Received: 4 May 2016 / Accepted: 13 June 2016 / Published online: 18 June 2016

(C) Springer-Verlag Berlin Heidelberg 2016

\begin{abstract}
Purpose The adoption of a sentinel lymph node (SLN) algorithm and the presence of high bilateral detection rates have been associated with increased accuracy of SLN mapping in cervical and endometrial cancer patients. In this context, the significance of the number of SLNs removed has not yet been investigated. The aim of this study was to evaluate (a) whether or not a higher SLN removal count is associated with a reduced false-negative rate and (b) which clinical factors correlate with the number of SLNs removed.

Methods Patients with cervical or endometrial cancer who underwent SLN mapping with bilateral SLN detection followed by lymphadenectomy were evaluated retrospectively. On the basis of the mean number of the SLNs removed, the patients were divided in two groups: Group 1 included patients with up to 3 SLNs removed and Group 2 included patients with more than 3 SLNs removed. Factors predicting a higher SLN count were evaluated using univariate and multivariate analysis.

Results Eighty-four patients met the inclusion criteria. The two groups consisted of 42 patients each and differed only by the median SLN count. Two endometrial cancer patients in Group 1 had false-negative pelvic SLNs and isolated para-aortic metastases; no false-negative SLNs were recorded in Group 2 ( $p=$ n.s.). The results of multivariate
\end{abstract}

Andrea Papadia

andrea.papadia@insel.ch

1 Department of Obstetrics and Gynecology, University Hospital of Berne and University of Berne, Effingerstrasse 102, 3010 Bern, Switzerland

2 Department of Gynecology and Obstetrics, Sapienza University of Rome, Rome, Italy analysis indicted that the number of SLNs removed was influenced only in cases where the operating surgeon had performed more than 20 laparoscopic ICG SLN mappings. Conclusions A higher SLN count does not seem to increase the accuracy of SLN mapping in cervical and endometrial cancer patients.

Keywords Endometrial cancer - Cervical cancer - Sentinel lymph node mapping · Indocyanine green · Sentinel lymph node count $\cdot$ Echelon lymph nodes

\section{Background}

Lymph nodal status is one of the most important prognostic factors driving adjuvant treatment of cervical and endometrial cancer. In early-stage cervical cancer, the presence of metastatic lymph nodal disease is regarded as a high-risk pathologic factor associated with a poorer survival rate and indicating the need for adjuvant chemoradiotherapy (Delgado et al. 1990; Peters et al. 2000). In endometrial cancer, despite the fact that two randomized trials failed to show any benefit of a lymphadenectomy, surgical staging was never abandoned, but rather tailored on the basis of intrauterine risk factors evaluated on intraoperative frozen section (Benedetti Panici et al. 2008; ASTEC Study Group et al. 2009; Kumar et al. 2012; Sala et al. 2014; Papadia et al. 2009; Morotti et al. 2012; Laufer et al. 2013). Although adjuvant treatment may be considered in node negative patients with pathologic risk factors in the uterus, such as LVSI, lymph nodal status is a fundamental factor in the decision to recommend further treatment after surgery (Simpkins et al. 2013; Colombo et al. 2016). Recently, NCCN guidelines have recognized SLN mapping as an acceptable alternative to surgical staging in clinically 
localized cervical and endometrial cancer patients (NCCN Guidelines 2015). Furthermore, the introduction of indocyanine green (ICG) as a tracer in conjunction with dedicated robotic and laparoscopic platforms seems to have significantly increased the bilateral detection rates and the "userfriendliness" of the SLN mapping in these cases (Imboden et al. 2015; Papadia et al. 2016; Tanner et al. 2015; How et al. 2015; Buda et al. 2016). We have previously reported high overall and bilateral detection rates and low false-positive rates in patients with cervical and endometrial cancer undergoing ICG SLN mapping (Imboden et al. 2015; Papadia et al. 2016).

Potential advantages of SLN mapping include the identification of lymph nodes located in unusual anatomic locations that would have not been removed otherwise and pathologic ultrastaging that allows for the identification of a small burden of metastatic disease that may have otherwise remained undetected. Furthermore, as opposed to a full lymphadenectomy, an SLN biopsy has been associated with reduced postoperative morbidity (Veronesi et al. 2003; Van der Zee et al. 2008). The single most important characteristic of the SLN is its false-negative rate, which has to be low in order to avoid the risk of undertreating a patient, thereby compromising her survival.

The SLN is defined as the first lymph node to receive lymphatic drainage from a tumor. However, especially if a combination of tracers (such as ${ }^{99} \mathrm{Tc}$ and blue dyes) or ICG (which is transported rapidly in the lymphatic vessels and has a long half-life) are used, multiple SLNs are often identified. Although additional lymph nodes other than the SLN should be considered as echelons, it is not always easy to distinguish clearly between the two. The removal of additional SLNs may reduce the benefit of the SLN mapping. On the other hand, the removal of all the lymph nodes draining the tracer may increase the safety of the procedure by reducing the false-negative rate.

The aim of the study is to evaluate whether or not a specific number of SLNs removed is associated with a reduced false-negative rate and which clinical factors correlate with the number of SLNs removed.

\section{Materials and methods}

A retrospective analysis of patients with complex atypical endometrial hyperplasia $(\mathrm{CAH})$ and clinical stage I and II cervical or endometrial cancer undergoing ICG SLN mapping at our Institution between December 2012 and January 2016 was conducted. Data on a subset of the patients in this cohort were previously published (Imboden et al. 2015; Papadia et al. 2016). Patients with CAH were included in the study because of the documented high risk of harboring endometrial cancer (Morotti et al. 2012). For the purpose of the study, only patients in whom the SLN mapping was successful bilaterally (patients who mapped in both hemipelvises) and in whom a pelvic lymph nodal dissection (PLND) and/or para-aortic lymph nodal dissection (PALND) was performed after having removed the SLNs were selected. Demographic, clinical, and pathologic data were retrieved retrospectively from an electronic database. Data on SLN mapping were collected prospectively. The study was IRB approved. All patients signed informed consent.

In every case, SLN mapping was performed as follows: after a diagnostic laparoscopy, the cervix was injected submucosally, one centimeter deep in the stroma at the four cardinal points, with a total of $8 \mathrm{ml}$ ICG. Two vials of $25 \mathrm{mg}$ ICG powder (Pulsion ${ }^{\circledR}$ ) had each been previously suspended with $5 \mathrm{ml}$ sterile water. After injection of the tracer, the pelvic peritoneum was opened and the camera mode was switched on NIR mode to detect the fluorescent signal and thereby the SLNs. Additionally, any clinically suspicious node was removed.

The management differed among patients with cervical cancer and patients with endometrial cancer or CAH. For cervical cancer patients, the SLNs removed as well as the clinically suspicious non-SLNs (NSLNs) were sent to frozen section analysis. If the SLNs and NSLNs removed were negative for metastatic disease, the planned surgical procedure was completed in patients with early-stage cervical cancer. If metastatic disease was involved in the SLNs and NSLNs that were removed, the radical procedure was aborted in favor of concurrent chemo-radiotherapy. In the latter cases, a laparoscopic para-aortic lymph node dissection was performed to determine the extension of the radiation fields.

For patients with endometrial cancer or $\mathrm{CAH}$, frozen section analysis of the SLNs was not performed routinely, but only if the SLNs appeared clinically suspicious for metastatic involvement. NSLNs that appeared macroscopically suspicious were removed and sent for frozen section. In the event of metastatic disease in the lymph nodes, a complete pelvic and para-aortic lymphadenectomy (PPALND) was performed. In the other cases, following the completion of the total laparoscopic hysterectomy, the uterus was sent to frozen section. Based on identification of tumor risk factors and clinical judgment, a laparoscopic PLND or PALND or both were performed. In every case, using final histopathological analysis, a complete ultrastaging of the SLNs was performed (three slides H\&E 200um, immunohistochemistry (IHC) if negative).

The false-positive rate was defined as zero. The overall detection rate was calculated by dividing the number of procedures in which at least one SLN was identified by the total number of procedures performed. A true-positive SLN was defined as a positive SLN identified with 
histopathological techniques (HE, serial sectioning, IHC), independent of regional lymph node status. A false-negative SLN mapping was defined as a bilateral negative SLN in combination with a metastatic NSLN.

Following the calculation of the median number of SLNs removed per patient, the cohort of patients was divided into two groups: Group 1 included patients with a lymph node count below the median number of removed SLNs; Group 2 included patients with a lymph node count above the median number of removed SLNs. Demographic and clinical characteristics of the two groups were compared using an unpaired $t$ test. The false-negative rates among the two groups were compared using Fischer's exact test. Univariate and multivariate analyses were performed in order to identify factors predicting a higher number of SLNs removed. Multivariable models were carried out for variables with a $p$ value $<0.2$ in univariate analysis. $p$ values $\leq 0.05$ were considered statistically significant. Statistical analysis was performed using IBM-Microsoft SPSS version 22.0.

\section{Results}

During the study period, 131 patients with $\mathrm{CAH}$, endometrial cancer, or cervical cancer underwent ICG SLN mapping. Data on overall and bilateral detection rates and false-negative rates were previously reported, separately for cervical and endometrial cancer patients from this cohort (Imboden et al. 2015; Papadia et al. 2016). Of these patients, 84 met study inclusion criteria (bilateral detection rate and full PPALND). The median number of sampled SLNs was 3.5 (range: 2-18). Based on this result, two groups of patients were created. Group 1 consisted of 42 patients with an SLN count of up to 3 lymph nodes and Group 2 consisted of 42 patients with a SLN count of 4 or more. Basic clinical and demographic characteristics did not differ between the two groups for age, BMI, type of cancer (cervical or endometrial), and mean pelvic and para-aortic NSLN count. The two groups differed only in the median SLN count ( 2 for Group 1 vs 5 for Group 2). Nine patients in Group 1 (21.5\%) and 12 patients in Group $2(28.6 \%)$ had metastatic SLNs $(p=$ n.s.). Eight patients in Group $1(19 \%)$ and 12 patients in Group 2 $(28.6 \%)$ had metastatic NSLNs in $(p=$ n.s.). Patient characteristics are summarized in Table 1.

In two endometrial cancer patients, both in Group 1, the SLNs were false negative. Two SLNs in one patient and three SLNs in the other patient were identified in the pelvis. In both patients, the SLNs and the pelvic NSLNs were negative, whereas one metastatic para-aortic NSLN was identified in one patient at the time of the final histopathological analysis, and two in the other patient. No false-negative SLNs were identified in Group 2. The SLN false-negative rate was 4.8 and $0 \%$ for Group 1 and Group 2, respectively
Table 1 Clinical and demographic characteristics of the patients

\begin{tabular}{|c|c|c|c|}
\hline Characteristics & Group $1(n=42)$ & Group $2(n=42)$ & $p$ \\
\hline Age (years) & $54.5(34-89)$ & $55(28-83)$ & 0.6 \\
\hline $\operatorname{BMI}\left(\mathrm{kg} / \mathrm{m}^{2}\right)$ & $25.6(17.4-43.6)$ & $44.9(19-40.2)$ & 0.8 \\
\hline Histologic diagnosis & & & 0.01 \\
\hline Endometrial cancer & $25(1 \mathrm{CS})$ & 20 & \\
\hline Cervical cancer & 17 & 22 & \\
\hline $\begin{array}{l}\text { Median number of } \\
\text { SLNs }\end{array}$ & $3(2-3)$ & $5(4-18)$ & $<0.001$ \\
\hline $\begin{array}{l}\text { Patients with meta- } \\
\text { static SLNs }\end{array}$ & $9(21.4 \%)$ & $12(28.6 \%)$ & 0.3 \\
\hline $\begin{array}{l}\text { Median number of } \\
\text { pelvic LN }\end{array}$ & 27 & 36 & 0.2 \\
\hline $\begin{array}{l}\text { Median number of } \\
\text { para-aortic LN }\end{array}$ & 12 (21 pts) & 20.5 (28 pts) & 0.21 \\
\hline $\begin{array}{l}\text { Pts with metastatic } \\
\text { pelvic NSLN }\end{array}$ & $8(19 \%)$ & $12(28.6 \%)$ & 0.17 \\
\hline $\begin{array}{l}\text { Metastatic para-aortic } \\
\text { NSLN }\end{array}$ & $6(14.3 \%)$ & $4(9.5 \%)$ & 0.24 \\
\hline
\end{tabular}

Patients in Group 1 and 2 differed only for median SLN count

Table 2 Impact on the number of SLNs removed on the false-negative rates

\begin{tabular}{lllll}
\hline $\begin{array}{l}\text { SLNs } \\
\text { removed } \\
(n)\end{array}$ & $\begin{array}{l}\text { Patients } \\
\text { with SLNs } \\
\text { identified } \\
(n)\end{array}$ & $\begin{array}{l}\text { Patients } \\
\text { with true- } \\
\text { positive } \\
\text { SLNs }(n)\end{array}$ & $\begin{array}{l}\text { Patients } \\
\text { with false- } \\
\text { negative } \\
\text { SLNs }(n)\end{array}$ & $\begin{array}{l}\text { False-nega- } \\
\text { tive rate (\%) }\end{array}$ \\
\hline$\leq 3$ & 42 & 9 & 2 & 4.8 \\
$>3$ & 42 & 12 & 0 & 0 \\
\hline
\end{tabular}

and did not differ significantly between the two groups (Table 2).

Patient characteristics (age, BMI), disease characteristics (type of tumor, presence of positive SLNs), and characteristics of the surgeon (experience of up to or over 20 ICG SLN and gynecologic oncology certification) were evaluated for influence on the SLN count. In the univariate analysis, the BMI of the patient, type of cancer (cervical vs endometrial), positive SLNs, surgical experience (up to 20 procedures vs over 20 procedures), and gynecologic oncology certification were considered significant and further analyzed at multivariate analysis. Only surgical expertise maintained significance as an independent prognostic factor for number of SLNs removed. Data on univariate and multivariate analysis are presented in Table 3.

\section{Discussion}

In the past decade, through the adoption of minimally invasive approaches and more recently through the acceptance 
Table 3 Univariate and multivariate analysis on factors possibly influencing SLN count

\begin{tabular}{|c|c|c|c|c|c|c|}
\hline & \multicolumn{3}{|c|}{ Univariate analysis } & \multicolumn{3}{|c|}{ Multivariate analysis } \\
\hline & $b(\mathrm{SE})$ & $\beta$ & $p$ & $b(\mathrm{SE})$ & $\beta$ & $p$ \\
\hline Age in years & $-0.026(0.021)$ & -0.131 & 0.234 & $0.004(0.027)$ & 0.021 & 0.876 \\
\hline $\mathrm{BMI}$ in $\mathrm{kg} / \mathrm{m}^{2}$ & $-0.083(0.052)$ & -0.175 & 0.112 & $-0.045(0.055)$ & -0.094 & 0.414 \\
\hline Type of cancer (endometrial vs cervical) & $1.188(0.674)$ & 0.191 & 0.081 & $0.596(0.875)$ & 0.096 & 0.498 \\
\hline Hx of pelvic surgery (yes vs no) & $0.005(0.834)$ & 0.001 & 0.995 & & & \\
\hline Surgical expertise ( $\leq$ vs $>20$ performed procedures) & $1.918(0.656)$ & 0.307 & 0.004 & $1.476(0.726)$ & 0.236 & 0.046 \\
\hline Gynecologic oncology certification (no vs yes) & $1.565(0.748)$ & 0.225 & 0.040 & $0.711(0.808)$ & 0.102 & 0.381 \\
\hline
\end{tabular}

Bold values indicates that significant for the multivariate analysis of $p$ value is less than 0.05

Age of the patient, BMI, type of tumor (endometrial vs cervical) and surgeon's expertise did not significantly influence the SLN count

of SLN mapping as an alternative to a full lymphadenectomy, the management of early-stage cervical and endometrial cancer has changed significantly (NCCN Guidelines 2015; Papadia et al. 2004; Walker et al. 2009; Ditto et al. 2015a, b). Since the introduction of ICG as a tracer and the availability of robotic and laparoscopic platforms for SLN mapping, this technique has gained widespread use, as documented in the large volume of literature on this topic. The majority of the literature deals with detection rates and false-negative rates of the SLN mapping (Imboden et al. 2015; Papadia et al. 2016; Tanner et al. 2015; How et al. 2015). We have shown previously that ICG SLN mapping yields higher bilateral detection rates than combined ${ }^{99} \mathrm{Tc}$ and patent blue SLN mapping in cervical cancer patients, with overall and bilateral detection rates of $95.5 \%$ (Imboden et al. 2015). In endometrial cancer, we recorded overall and bilateral detection rates of 96 and $88 \%$, respectively, in 75 patients undergoing ICG SLN mapping (Papadia et al. 2016). However, no one has focused thus far on how many SLNs need to be removed for the mapping to be oncologically safe.

In our series, the median number of SLNs removed per patient was 3.5. The SLNs were false negative in two patients with endometrial cancer. These data compare favorably with those of Barlin et al., who reported a decline in their false-negative rate from 15 to $2 \%$ after applying an SLN mapping algorithm (Barlin et al. 2012). Furthermore, the false-negative SLN rate did not differ significantly between the groups of patients with a lower or higher SLN count. Interestingly, the two patients in whom the falsenegative SLNs were identified had isolated para-aortic metastases, in one and two lymph nodes, respectively, with negative pelvic SLNs and negative pelvic NSLN. These data are in line with the risk of isolated para-aortic metastasis reported by Mariani el al in fully staged high-risk EMCA patients (Kumar et al. 2014). In these cases, sampling a larger number of nodes would have probably not led to the identification of these metastatic lymph nodes. It is unclear whether or not a hysteroscopic peritumoral tracer injection would have facilitated the identification of these lymph nodes; so far, the cervical tracer injection remains the preferred method for SLN mapping in endometrial cancer (Ditto et al. 2015a, b; Bogani et al. 2015).

When performing a SLN biopsy, the surgeon has to weigh the risks of dissecting as many lymph nodes as in the case of a full lymphadenectomy against the risk of missing a metastatic lymph node. One of the characteristics of ICG is that it travels relatively quickly through the lymphatic vessels. However, in comparison with blue dyes, the detection of which practically disappears after $30 \mathrm{~min}$, the fluorescent signal of ICG remains in the lymph nodes for a longer time. This may lead to the identification of several fluorescent lymph nodes, and at times it may be difficult to distinguish between a true SLN that is draining an independent lymphatic vessel and an echelon lymph node that has drained the tracer after it travelled through a real SLN. In cases when a significant amount of time elapses between the intracervical injection and the retroperitoneal exploration, such as when a prolonged adhesiolysis is needed, multiple lymph nodes may appear fluorescent.

The question of how many SLNs to remove is not a new one. This topic has been investigated in breast cancer surgery with heterogeneous results. It is recommended that after ${ }^{99} \mathrm{Tc}$ tracer injection, every SLN with a CPM of more than $10 \%$ of the "hottest" node be removed (Martin et al. 2000). Some authors suggested that when a combination of ${ }^{99} \mathrm{Tc}$ and blue dyes is used, removing more than four SLNs does not impact significantly on the accuracy of the mapping (Zakaria et al. 2007; Goyal et al. 2006; McCarter et al. 2001; Woznick et al. 2006; Chagpar et al. 2007; Vaidya et al. 2005). In breast cancer surgery, the removal of five or more SLNs is associated with higher rates of axillary seroma and wound infection (Wilke et al. 2006). 
It is common that more than one SLN is identified in each patient. In a multicenter validation trial in melanoma, an average of 2.8, 2.3 and 2.6 SLNs were removed from the axilla, groin and neck, respectively (Morton et al. 1999). This may reflect the passing through of dye from the "true" SLN into secondary echelon nodes, or simply normal anatomical variation in which the lymphatics of a given site drain simultaneously to more than one SLN.

Our results indicate that removal of more than 3 sentinel lymph nodes does not affect the accuracy of the SLN mapping. Therefore, we do not want to set an upper limit to the number of SLNs that should be sampled, but rather highlight the fact that removing all stained lymph nodes may not necessarily improve the accuracy of the SLN mapping. ICG has a long half-life and remains in the lymphatic system for a long time. If we wait long enough after tracer injection, the SLNs and the echelons lymph nodes draining the SLNs will all uptake the tracer and stain fluorescent. In these cases, an SLN mapping may translate easily into a focused sampling or even into a complete lymphadenectomy.

We then tried to assess the influence of a patient characteristics (age, BMI), tumor characteristics (type of tumor, presence of metastatic SLNs), and the surgeon's expertise (gynecologic oncology certification, number of procedures performed) on the number of removed SLNs using univariate and multivariate analysis. In multivariate analysis, the number of removed SLNs correlated only with the number (more that 20) of laparoscopic ICG SLN mappings performed by the operating surgeon. Gynecologic oncology certification was not an independent variable affecting SLN count. With greater specific experience in ICG SLN mapping, surgeons may be more confident in their ability to correctly discern SLNs from echelon nodes, leading to the removal of a smaller number of nodes. These data correlate well with what is considered to be the learning curve for SLN mapping in breast cancer (McMasters et al. 2001).

Other factors that may influence the number of SLNs identified are the concentration and volume of ICG injected. In our institution, we inject a volume of $8 \mathrm{ml}$ of ICG solution at a concentration of $5 \mathrm{mg} / \mathrm{ml}$ for a total of $40 \mathrm{mg}$ of ICG. This dose is significantly higher than the dose used by other authors, who typically inject a total of $2 \mathrm{ml}$ at a concentration of $1.25 \mathrm{mg} / \mathrm{ml}$ (Ruscito et al. 2016; Buda et al. 2016). We were unfortunately unable to assess the influence of the volume and concentration of ICG solution injected on the number of SLNs removed, since the amount of ICG solution used in every patient was identical.

Limitations of the study include its retrospective nature and the relatively small sample size. On the other hand, the prospective SLN mapping data collection and the inclusion of patients who mapped bilaterally only and were surgically staged corroborate the strengths of the study. To our knowledge, this is the first series that seeks to identify an optimal number of SLNs removed. Although for every SLN that drains, an independent lymphatic vessel should be sampled. In our experience, removing more than 3 SLNs did not increase the accuracy of the SLN mapping.

\section{Compliance with ethical standards}

Conflict of interest None of the authors has any conflict of interest.

\section{References}

ASTEC Study Group, Kitchener H, Swart AM, Quian Q, Amos C, Parmar MK (2009) Efficacy of systematic pelvic lymphadenectomy in endometrial cancer (MRC ASTEC trial): a randomised study. Lancet 373:125-136

Barlin JN, Khoury-Collado F, Kim CH, Leitao MM, Chi DS, Sonoda $\mathrm{Y}$ et al (2012) The importance of applying a sentinel lymph node mapping algorithm in endometrial cancer staging: beyond removal of blue nodes. Gynecol Oncol 125:531-535

Benedetti Panici P, Basile S, Maneschi F, Lissoni A, Signorelli M, Scambia G et al (2008) Systematic pelvic lymphadenectomy vs. no lymphadenectomy in early-stage endometrial carcinoma: randomized clinical trial. J Natl Cancer Inst 100:1707-1716

Bogani G, Ditto A, Martinelli F, Signorelli M, Perotto S, Lorusso D et al (2015) A critical assessment on the role of sentinel node mapping in endometrial cancer. J Gynecol Oncol. 26:252-254

Buda A, Papadia A, Zapardiel I, Vizza E, Ghezzi F, De Ponti E et al (2016) From conventional radiotracer Tc-99-m with blue dye to indocyanine green fluorescence: a comparison of methods towards optimization of sentinel lymph node mapping in early stage cervical cancer for a laparoscopic approach. Ann Surg Oncol PMID: 27126631

Chagpar AB, Scoggins CR, Martin RC, Carlson DJ, Laidley AL, ElEid SE et al (2007) Are 3 sentinel nodes sufficient? Arch Surg 142:456-460

Colombo N, Creutzberg C, Amant F, Bosse T, Gonzaléz-Martin A, Lederman J, Marth C et al (2016) ESMO-ESGO-ESTRO consensus conference on endometiral cancer diagnosis, treatment and follow up. Int J Gynaecol Cancer 26:2-30

Delgado G, Bundy B, Zaino R, Sevin BU, Creasman WT, Major F (1990) Prospective surgical-pathological study of disease-free interval on patients with stage IB squamous cell carcinoma of the cervix: a Gynecologic Oncology Group study. Gynecol Oncol 38:352-357

Ditto A, Martinelli F, Bogani G, Gasparri ML, Di Donato V, Zanaboni $F$ et al (2015a) Implementation of laparoscopic approach for type B radical hysterectomy: a comparison with open surgical operations. Eur J Surg Oncol 41:34-39

Ditto A, Martinelli F, Bogani G, Papadia A, Lorusso D, Raspagliesi F (2015b) Sentinel node mapping using hysteroscopic injection of indocyanine green and laparoscopic near-infrared fluorescence imaging in endometrial cancer staging. J Minim Invasive Gynecol 22:132-133

Goyal A, Newcombe RG, Chhabra A, Mansel RE, ALMANAC Trialist Group (2006) Factors affecting failed localization and falsenegative rates of sentinel node biopsy in breast cancer-results of the ALMANAC validation phase. Breast Cancer Res Treat 99:203-208

How J, Gotlieb WH, Press JZ, Abitbol J, Pelmus M, Ferenczy A et al (2015) Comparing indocyanine green, technetium, and blue dye 
for sentinel lymph node mapping in endometrial cancer. Gynecol Oncol 137:436-442

Imboden S, Papadia A, Nauwerk M, McKinnon B, Kollman Z, Mohr $S$ et al (2015) A comparison of radiocolloid and indocyanine green fluorescence imaging, sentinel lymph node mapping in patients with cervical cancer undergoing laparoscopic surgery. Ann Surg Oncol 22:4198-4203

Kumar S, Medeiros F, Dowdy SC, Keeney GL, Bakkum-Gamez JN, Podratz KC et al (2012) A prospective assessment of the reliability of frozen section to direct intraoperative decision making in endometrial cancer. Gynecol Oncol 127:525-531

Kumar S, Podratz KC, Bakkum-Gamez JN, Dowdy SC, Weaver AL, McGree ME et al (2014) Prospective assessment of the prevalence of pelvic, paraaortic and high paraaortic lymph node metastasis in endometrial cancer. Gynecol Oncol 132:38-43

Laufer J, Scasso S, Papadia A, Sosa C, Cirillo F, Raspagliesi F (2013) Association between tumor diameter and lymphovascular space invasion among women with early-stage endometrial cancer. Int J Gynaecol Obstet 123:142-145

Martin RC, Edwards MJ, Wong SL, Tuttle TM, Carlson DJ, Brown CM et al (2000) Practical guidelines for optimal gamma probe detection of sentinel lymph nodes in breast cancer: results of a multiinstitutional study. Surgery 128:139-144

McCarter MD, Yeung H, Fey J, Borgen PI, Cody HS (2001) The breast cancer patient with multiple sentinel nodes: when to stop? J Am Coll Surg 192:692-697

McMasters KM, Wong SL, Chao C, Woo C, Tuttle TM, Noyes RD et al (2001) Defining the optimal surgeon experience for breast cancer sentinel lymph node biopsy: a model for implementation of new surgical techniques. Ann Surg 234:292-300

Morotti M, Menada MV, Moioli M, Sala P, Maffeo I, Abete L et al (2012) Frozen section pathology at time of hysterectomy accurately predicts endometrial cancer in patients with preoperative diagnosis of atypical endometrial hyperplasia. Gynecol Oncol 125:536-540

Morton DL, Thompson JF, Essner R, Elashoff R, Stern SL, Nieweg OE et al (1999) Validation of the accuracy of intraoperative lymphatic mapping and sentinel lymphadenectomy for early-stage melanoma: a multicenter trial. Multicenter Selective Lymphadenectomy Trial Group. Ann Surg 230:453-463

Papadia A, Remorgida V, Salom Ragni N (2004) Laparoscopic lymphadenectomy in gynecologic oncology: a review. J Am Assoc Gynecol Laparosc 11:297-306

Papadia A, Azioni G, Brusacà B, Fulcheri E, Nishida K, Menoni S et al (2009) Frozen section underestimates the need for surgical staging in endometrial cancer patients. Int J Gynecol Cancer 19:1570-1573

Papadia A, Imboden S, Siegenthaler F, Gasparri ML, Mohr S, Lanz S et al (2016) Laparoscopic Indocyanine green sentinel lymph node mapping in endometrial cancer. Ann Surg Oncol 23:2206-2211
Peters WA, Liu PY, Barrett RJ, Stock RJ, Monk BJ, Berek JS et al (2000) Concurrent chemotherapy and pelvic radiation therapy compared with pelvic radiation therapy alone as adjuvant therapy after radical surgery in high-risk early-stage cancer of the cervix. J Clin Oncol 18:1606-1613

Ruscito I, Gasparri ML, Braicu EI, Bellati F, Raio L, Sehouli J et al (2016) Sentinel node mapping in cervical and endometrial cancer: indocyanine green versus other conventional dyes - a metaanalysis. Ann Surg Oncol PMID

Sala P, Morotti M, Menada MV, Cannavino E, Maffeo I, Abete L et al (2014) Intraoperative frozen section risk assessment accurately tailors the surgical staging in patients affected by early-stage endometrial cancer: the application of 2 different risk algorithms. Int J Gynecol Cancer 24:1021-1026

Simpkins F, Papadia A, Kunos C, Michener C, Frasure H, AbuShahin F et al (2013) Patterns of recurrence in stage I endometrioid endometrial adenocarcinoma with lymphovascular space invasion. Int J Gynecol Cancer 23:98-104

Tanner EJ, Sinno AK, Stone RL, Stone RL, Levinson KL, Long KC et al (2015) Factors associated with successful bilateral sentinel lymph node mapping in endometrial cancer. Gynecol Oncol 138:542-547

Vaidya JS, Dewar JA, Brown DC, Thompson AM (2005) A mathematical model for the effect of a false-negative sentinel node biopsy on breast cancer mortality: a tool for everyday use. Breast Cancer Res 7:225-227

Van der Zee AG, Oonk MH, De Hullu JA, Ansink JA, Vergote I, Verheijen RH, Maggioni A et al (2008) Sentinel node dissection is safe in the treatment of early stage vulvar cancer. J Clin Oncol 26:884-889

Veronesi U, Paganelli G, Viale G, Luini A, Zurrida S, Galimberti V et al (2003) A randomized comparison of sentinel-node biopsy with routine axillary dissection in breast cancer. $\mathrm{N}$ Engl $\mathrm{J}$ Med 349:546-553

Version 2.2015: NCCN Clinical Practice Guidelines in Oncology

Walker JL, Piedmonte MR, Spirtos NM, Eisenkop SM, Schlaerth JB, Mannel RS et al (2009) Laparoscopy compared with laparotomy for comprehensive surgical staging of uterine cancer: gynecologic Oncology Group Study LAP2. J Clin Oncol 27:5331-5336

Wilke LG, McCall LM, Posther KE, Whitworth PW, Reintgen DS, Leitch AM et al (2006) Surgical complications associated with sentinel lymph node biopsy: results from a prospective international cooperative group trial. Ann Surg Oncol 13:491-500

Wong SL, Edwards MJ, Chao C, Tuttle TM, Noyes RD, Carlson DJ et al (2001) Sentinel lymph node biopsy for breast cancer: impact on the number of sentinel lymph nodes removed on the false negative rate. J Am Coll Surg 192:684-689

Woznick A, Franco M, Bendick P, Benitez PR (2006) Sentinel lymph node dissection for breast cancer: how many nodes are enough and which technique is optimal? Am J Surg 191:330-333

Zakaria S, Degnim AC, Kleer CG, Diehl KA, Cimmino VM, Chang AE et al (2007) Sentinel lymph node biopsy for breast cancer: how many lymph nodes are enough? J Surg Oncol 96:554-559 